\title{
La práctica logopédica en los servicios comunitarios desde la formación docente
}

\section{Speech therapy practice in community services from teacher training}

\author{
Juana Emilia Bert Valdespino ${ }^{1}$ \\ jbertva@gmail.com \\ https://orcid.org/0000-0002-9990-7854 \\ Irina Naranjo Bert ${ }^{2}$ \\ vinculacionits@sudamericano.edu.ec \\ https://orcid.org/0000-0001-8356-4853 \\ Alina Rodríguez Morales ${ }^{3}$ \\ alina.rodriguezm@ug.edu.ec \\ https://orcid.org/0000-0003-3714-2638
}

Recibido: 28/6/2021, Aceptado: 28/9/2021

\begin{abstract}
RESUMEN
La Universidad Nacional de Educación (UNAE) asume el reto de contribuir con la formación de profesionales que se ocupen de la atención a los trastornos del lenguaje, habla, voz y de la comunicación en general en las instituciones educativas y especializadas en Ecuador. Para ello cuenta con el itinerario de Logopedia en la carrera de Educación Especial, la cual, forma educadores con competencias humanas y profesionales comprometidas con la atención a la diversidad siguiendo los preceptos y compromisos de la educación inclusiva. Este artículo tiene el propósito de reflexionar sobre algunas experiencias que evidencian la formación de tales competencias en el itinerario, sustentadas en el Modelo Pedagógico de la universidad. Se emplearon como métodos la investigación acción, la observación participante, el análisis de contenido, análisis del producto de la actividad, la encuesta y la entrevista abierta a los estudiantes del $7 \mathrm{mo}$ ciclo de la carrera. Los resultados evidencian el tránsito de las inquietudes y angustias al desarrollo de las capacidades para la prevención, el diagnóstico y la intervención de los trastornos del habla por los estudiantes.
\end{abstract}

Palabras clave: docencia universitaria, educación especial, UNAE.

\section{ABSTRACT}

The National University of Education (UNAE) takes on the challenge of contributing to the training of professionals who deal with the care of language, speech, voice and communication disorders in general in educational and specialized institutions

\footnotetext{
${ }^{1}$ Doctora en Educación, Universidad Nacional de Educación, Ecuador.

${ }^{2}$ Magíster, Instituto Superior Tecnológico Particular Sudamericano de Cuenca, Ecuador.

${ }^{3}$ Doctora, Universidad de Guayaquil, Ecuador.
} 


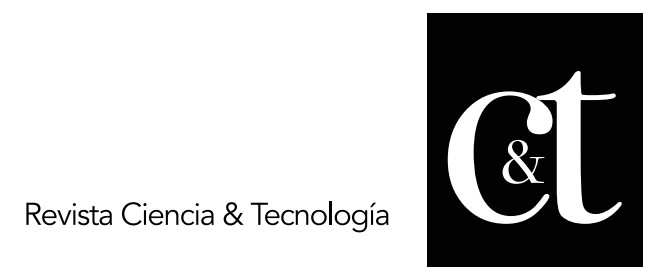

No. 32, 31 de octubre de 2021

ISSN impreso: 1390 - 6321

ISSN online: 2661 - 6734

in Ecuador. For this, it has the Speech Therapy itinerary in the Special Education career, which trains educators with human and professional skills committed to attending to diversity, following the precepts and commitments of inclusive education. This article has the purpose of reflecting on some experiences that show the formation of such competencies in the itinerary, based on the Pedagogical Model of the university. Action research, participant observation, content analysis, activity product analysis, survey and open interview with students in the 7th cycle of the degree were used as methods. The results show the transition from concerns and anxieties to the development of capacities for the prevention, diagnosis and intervention of speech disorders by students.

Keywords: University teaching, special education, UNAE

\section{Introducción}

La atención a la diversidad desde los preceptos de la educación inclusiva implica tomar en cuenta las particularidades individuales de cada niño, adolescente y joven que se forma en las instituciones educativas, sin embargo, hasta hoy en Ecuador resulta insuficiente el trabajo que se realiza en estos centros con los educandos que presentan trastornos en el lenguaje, lo cual se debe en gran medida, a que los estudios relacionados con la especialidad se asumen generalmente, por las ciencias médicas y no por las pedagógicas.

En una compilación de actas del XXV Congreso de Logopedia, Foniatría y Audiología (2006) se recoge que la formación profesional de los especialistas que atienden los trastornos del lenguaje a nivel internacional es muy diversa, lo que se refleja en una nomenclatura no uniforme de la profesión. Igualmente varían los niveles académicos y los campos de formación en los diferentes países. En América Latina, en cuanto a los términos se usan alrededor cuatro: logopeda, fonoaudiólogo, terapista del lenguaje, y tecnólogo médico especialista en trastornos del lenguaje. Estos tres últimos son los que confluyen en Ecuador.

La dispersión en la formación de estos profesionales refleja la diversidad de criterios y políticas que asumen los estados a la hora de atender los trastornos en el área de la comunicación y el lenguaje, prevaleciendo a nivel internacional un enfoque clínico que limita el compromiso por cubrir la atención a estos trastornos en los centros escolares, lo que afecta tanto la formación de los especialistas desde las ciencias de la educación, como la preparación de los docentes para que contribuyan desde un proceso enseñanza-aprendizaje inclusivo, con la prevención y atención de estos escolares.

En este contexto, la UNAE comprometida con la transformación de la educación ecuatoriana para lograr la formación integral de la diversidad de educandos en igual de oportunidades, asume el reto de contribuir con la formación de profesionales que se ocupen de la atención a los trastornos del lenguaje, habla, voz para que ejerzan sus funciones en las instituciones educativas regulares y especializadas de Ecuador, apoyando los procesos de aprendizaje y la inclusión socioeducativa de esta población infantojuvenil. 


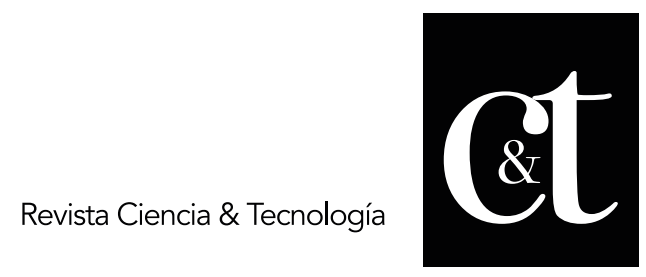

No. 32, 31 de octubre de 2021

ISSN impreso: 1390 - 6321

ISSN online: 2661 - 6734

El modelo pedagógico de la UNAE en sus fundamentos epistemológicos significa la formación integral de los docentes, que implica conocimientos, habilidades, emociones, actitudes y valores, en los que se involucran el saber pensar, saber decir y saber hacer, desde estas perspectivas en esta universidad se forman estudiantes en el itinerario de logopedia con competencias para problematizar las distintas situaciones que surjan en su especialidad, analizarlas, diagnosticarlas, proponer y evaluar procesos de mejoras a partir de un pensamiento investigativo e innovador.

Entre los principios que se destacan en este modelo de formación profesional guiaron el presente trabajo: el aprender haciendo; el currículo basado en casos, problemas y proyectos; la metacognición y la función tutorial del docente; los que orientan la relevancia del componente práctico en relación dialéctica con la teoría y mediatizado por la investigación para comprender, cuestionar, construir el nuevo saber y diseñar propuestas que resuelvan desde este binomio los problemas auténticos que se dan en los contextos educativos donde desarrollar las prácticas pre profesionales los estudiantes.

Dentro de las orientaciones metodológicas de la carrera de Educación Especial de la UNAE para resolver los problemas de la profesión se estacan la investigación en la acción, experimentando y reflexionando los casos, situaciones y problemas que se presentan en las instituciones educativas en la práctica pre profesional que asumen desde el primer ciclo. Una investigación formativa que desde la apropiación de los saberes disciplinares deviene en proyectos integradores de saberes en los que se proponen y aplican propuestas de mejora a partir de diagnosticar la realidad educativa, construyendo un conocimiento y un pensamiento innovador y creativo (Herrera, JI; Parrilla, A; Blanco, A; Y Guevara, G; 2018)

A decir de Bert, J; Herrera JI; Guevara, G (2019) "El proceso de implementación de la carrera permite ofrecer como principales hallazgos la articulación de la teoría y la práctica en un proceso inductivo- deductivo donde los estudiantes pueden vincular coherentemente el componente académico del currículo con acercamientos a la realidad de las instituciones educativas a través de las prácticas preprofesionales de manera sistemática, que se completa con procesos investigativos eminentemente cualitativos en función del logro de competencias docentes para asumir la inclusión educativa." (pág. 9)

En particular, el itinerario de logopedia sustentado en los postulados teóricos, metodológicos y prácticos del Modelo Pedagógico de la UNAE y de la carrera de educación especial, constituye parte del campo de formación profesional en $7 \mathrm{mo}$ ciclo y de la unidad de titulación en 8 vo y 9 no y, tiene sus antecedentes formativos en las asignaturas relacionadas con fundamentos neurológicos, psicológicos, pedagógicos y sociológicos que se desarrollan en el campo de formación básica en los primeros ciclos. 


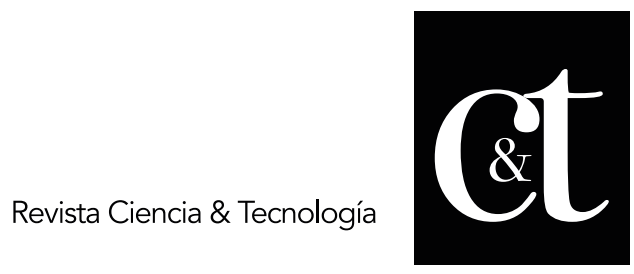

No. 32, 31 de octubre de 2021

ISSN impreso: 1390 - 6321

ISSN online: 2661 - 6734

Desde el itinerario se desarrollan los contenidos conceptuales y procedimentales a partir de un currículo interdisciplinario que articula coherentemente los espacios académicos investigativos y la vinculación con la sociedad, mediante las prácticas pre profesionales y las actividades de servicios comunitarios, en las que, desde una condición situada desarrollan capacidades para sumir los procesos de prevención, evaluación, diagnóstico e intervención de los trastornos del lenguaje, del habla, la voz y la comunicación en general de niños adolescentes y jóvenes con y sin discapacidad, con altos valores inclusivos.

El aprender y desarrollar capacidades directamente en los contextos educativos reales de las instituciones ecuatorianas desde un constante ir y venir de la teoría a la práctica y viceversa, implica que en los estudiantes surjan inquietudes, incertidumbre, angustias y cuestionamientos que se constituyen en contradicciones que guían los procesos de reflexión, problematización y construcción del conocimiento. De ahí que el objetivo de éste trabajo es: Reflexionar sobre las experiencias vividas durante el presente $7 \mathrm{mo}$ y $8 \mathrm{vo}$ ciclo de los estudiantes del itinerario de Logopedia de la carrera de Educación Especial de la UNAE, en el desarrollo de capacidades para comprender, diagnosticar, diseñar y evaluar los trastornos del lenguaje mediante la formación de competencias profesionales y humanas los comprometidas con procesos de inclusión educativa de la diversidad en el contexto ecuatoriano.

\section{Material y Métodos}

El presente trabajo se asume desde un estudio cualitativo que surge a partir de las experiencias transitadas con los estudiantes de $7 \mathrm{mo}$ durante el ciclo académico abril-agosto 2021. La unidad de análisis estuvo conformada por 24 estudiantes del itinerario de logopedia, 20 alumnos con trastornos en el lenguaje del centro especializado Unidad de Diagnóstico, Investigación Psicopedagógica y Apoyo a la Inclusión (UDIPSAI) y sus familias. La estrategia metodológica seguida es la investigación acción, complementada con los métodos observación participante, análisis de contenido, análisis del producto de la actividad, encuesta, entrevista abierta, datos fotográficos, grabaciones en vídeo, diario de campo y técnicas logopédicas no convencionales.

El trabajo siguiendo la investigación acción se despliega durante 4 etapas fundamentales: planificación, acción, observación y reflexión como una espiral dialéctica, las que transcurrieron durante el ciclo académico.

\section{Primera etapa: Planificación- Diagnóstico}

Durante esta etapa se diseñó el Syllabus y el plan de prácticas pre-profesionales de conjunto con los estudiantes. Los contenidos en ambos guardan una interrelación dialéctica en el entramado de los componentes teóricos-prácticosinvestigativos articulados en los núcleos teóricos y ejes integradores de ambos ciclos. Todo lo cual colocó a los estudiantes en el centro del proceso de aprendizaje y los orientó en las posibles temáticas que podían asumir para desarrollar sus proyectos integradores de saberes, dentro del marco de las prácticas pre profesionales. Se realizaron reuniones con los directivos de UDIPSAI para organizar los procesos de las prácticas pre profesionales en estas instituciones. 


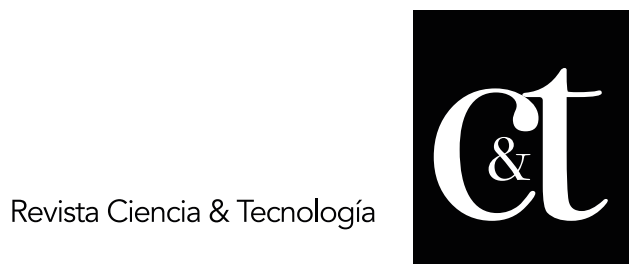

No. 32, 31 de octubre de 2021

ISSN impreso: 1390 - 6321

ISSN online: 2661 - 6734

Se desarrolló el proceso de inducción con los directivos, docentes y especialistas donde se socializa y enriquece el plan de práctica pre profesionales unido al de vinculación con la sociedad, se pone a los centros de prácticas en consonancia con los preceptos y contenidos del itinerario desde el Modelo Pedagógico de la UNAE y se tomaron los acuerdos para una adecuada organización del proceso. Se realizó un taller de preparación e intercambio a los estudiantes por parte de los logopedas y docentes de ambas instituciones donde se caracterizaron los alumnos con los que iban a trabajar, se dieron las pautas de atención desde el Modelo de gestión de éstas, se recogieron y solventaron las inquietudes de los estudiantes. Igualmente, los estudiantes hicieron un análisis documental del Modelo de Gestión de ambas instituciones desde una mirada crítica y reflexiva encontrando fortalezas, las que asumieron para su trabajo y barreras que frenan los procesos inclusivos, para las que propusieron acciones de mejora a partir de un estudio de los recursos y apoyos que se encuentran en estos centros y en sus contextos comunitarios, los que igualmente serían aprovechados por ellos en su intervención logopédica con los casos, todo lo cual a partir de los estudios teóricos que iban sustentando los hallazgos prácticos.

Durante esta etapa las tutoras académicas desarrollaron 3 tutorías de acompañamiento y académicas grupales para solventar dudas y una mejor orientación del proceso formativo mediante las prácticas pre profesionales y las actividades de vinculación con la sociedad, las que daban continuidad al trabajo. Se aplicó una encuesta a los estudiantes en la que, el 96\% evidenció inquietudes, angustias e incertidumbre ante el tratamiento logopédico y psicopedagógico que debían asumir directamente con los alumnos de estos centros, ya que asumirían trastornos que irían estudiando por primera vez en un proceso formativo que articula teoría práctica, lo cual estuvo igualmente mediado por una alta motivación e interés mostrada en el $100 \%$ de los estudiantes.

A partir de estos primeros momentos se realizaron los ajustes al plan de prácticas pre profesionales, al de vinculación con la sociedad y, a los contenidos del syllabus de las asignaturas de la práctica, del itinerario de logopedia y de Cátedra Integradora, desde un carácter flexible del currículo y buscando el ajuste a las sugerencias e inquietudes dadas por los estudiantes, logrando una mejor contextualización, articulación y la respuesta a las particularidades psicopedagógicas de éstos. Los estudiantes se organizaron en parejas pedagógicas siguiendo los preceptos del modelo pedagógico y se les entregaron los alumnos con trastornos del habla y del lenguaje con los que iban a trabajar.

Los estudiantes mediante la observación participante en los primeros encuentros con los alumnos de las dos instituciones de las prácticas pre profesionales en sus hogares y aulas, identificaron las situaciones problemáticas que asumieron para desarrollar sus PIENSA en el caso de los de $7 \mathrm{mo}$ ciclo y para el trabajo de titulación los de $8 \mathrm{vo}$ ciclo en el contexto de la intervención logopédica y psicopedagógica que desarrollaron con los alumnos de UDIPSAI y la EE Tamarit. A partir de aquí elaboraron el diseño teórico-metodológico que les orientó en el proceso académico de investigación acción a desarrollar. Bajo el acompañamiento de las tutoras académicas y las orientaciones dadas por éstas en las guías didácticas, los estudiantes diseñaron los instrumentos de recogida de información. 


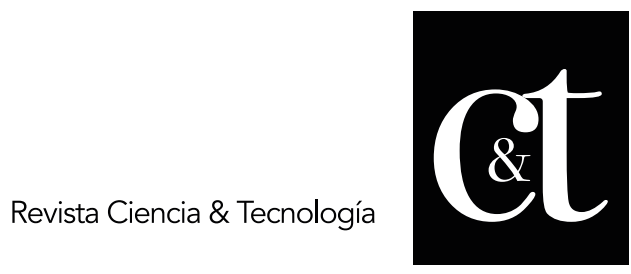

No. 32, 31 de octubre de 2021

ISSN impreso: 1390 - 6321

ISSN online: 2661 - 6734

Igualmente, realizaron una revisión documental a los expedientes psicopedagógicos y fichas logopédicas de los alumnos, aplicaron una entrevista a las familias de éstos mediante una ficha psicosocial elaborada por los estudiantes. Igualmente diseñaron y aplicaron una entrevista a las especialistas y docentes de los casos en estudio.

A partir del estudio de técnicas logopédicas no convencionales asumieron las que consideraron necesarias aplicar a sus alumnos bajo la asesoría y acompañamiento de las tutoras académicas y profesionales en clases y en tutorías. Realizaron el procesamiento de la información de todos los instrumentos aplicados, obteniendo una caracterización integral actualizada de los casos quedando evidenciadas las necesidades educativas actualizadas en el área del lenguaje y habla a partir de enriquecer y actualizar el diagnóstico logopédico, ya dado por terapistas del lenguaje; así mismo caracterizaron los procesos cognitivos, afectivos y psicomotriz de los alumnos e identificaron las barreras para el aprendizaje, la participación y desarrollo de la comunicación de los casos en estudio.

\section{Segunda etapa: Plan de Intervención-acción}

A partir del diagnóstico los estudiantes elaboraron el diseño teórico-metodológico de los Proyectos Integradores de Saberes (PIENSA) y los planes de mejora que se concretaron en las Estrategias de Intervención logopédica y psicopedagógicas con enfoque integral e inclusivo, en el marco de los procesos de investigación acción en los que estaban desarrollando su aprendizaje. A partir de la estrategia planificaron los tratamientos logopédicos, que contaban con ejercicios terapéuticos, funcionales, actividades, recursos, materiales y evaluaciones, desde un pensamiento innovador y creativo que les permitió atender los diferentes componentes del lenguaje afectados, el resto de las áreas de desarrollo y encausar el trabajo de superación de las barreras con la participación de los docentes tutores profesionales y las familias. Así mismo durante esta etapa realizaron estudios teóricos sobre los elementos teóricos-conceptuales y los antecedentes de la problemática identificada que les permitió un mayor nivel de profundización en los fundamentos anátomofisiológicos, pedagógicos, sociológicos de los trastornos del habla y del lenguaje de los alumnos de UDIPSAI con los que iban desarrollando sus prácticas pre profesionales.

\section{Tercera etapa: Observación-Reflexión}

Durante esta etapa se desarrollaron los tratamientos logopédicos tanto individuales en sus hogares, como en los grupos clase de los casos con trastornos del habla y del lenguaje, ambos a través de la plataforma zoom. En el transcurso de este proceso de atención directa se privilegiaron la observación participante en dos direcciones: de los estudiantes a los casos que atendían y de los docentes tutores académicos a los estudiantes en sus desempeños. La reflexión se desarrolló sistemáticamente durante las clases de las asignaturas de la práctica pre profesional y Cátedra Integradora integrando los aportes conceptuales y procedimentales del itinerario de Logopedia y del resto de las materias del ciclo, transitando de la vía inductiva a la deductiva, de la práctica y la vivencia a la teoría, utilizando los resultados de la observación participante y el diario de campo, desde un enfoque cíclico y dialéctico de investigación acción, en las que se analizaron los indicadores en estudio sobre los logros que se iban alcanzando y las 


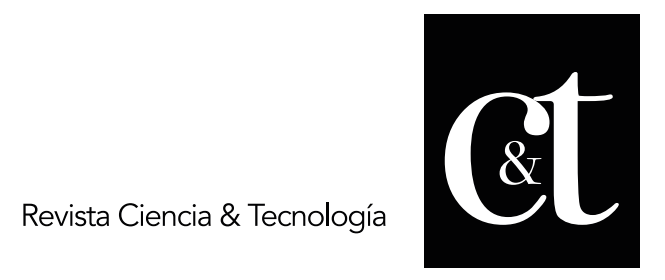

No. 32, 31 de octubre de 2021

ISSN impreso: 1390 - 6321

ISSN online: 2661 - 6734

dificultades que se constituían en barreras para el progreso de los casos. Durante esta etapa se realizaron, además, 4 talleres de reflexión, intercambio, retroalimentación con los estudiantes en los que exponían las planificaciones de los tratamientos, el desarrollo de éstos donde presentaban videos y fotos para evidenciarlos y analizarlos de conjunto con las tutoras académicas y las tutoras profesionales.

La autorreflexión realizada por los estudiantes mediante la autoevaluación desde un enfoque metacognitivo, contribuyó junto a la coevaluación y la heteroevaluación sistemática, todo lo cual guiado por rúbricas evaluativas permitieron procesos de mejoras de los tratamientos, el desarrollo de las capacidades para la prevención, el diagnóstico y la intervención que iban desarrollando con los casos y logros en los alumnos con trastornos en el habla y el lenguaje.

\section{Cuarta etapa: Evaluación-Propuestas de mejora}

Una vez culminada las etapas anteriores, se realizaron procesos evaluativos que contemplaron los resultados de las evaluaciones sistemáticas realizadas durante éstas y mediante talleres de evaluación, participación y retroalimentación los estudiantes presentaron un informe psicopedagógico de cada caso que recogía los resultados alcanzados en relación al trastorno del habla o del lenguaje y en cada área de desarrollo trabajada en sus alumnos. Se analizaron los logros a partir del diagnóstico dado en la primera etapa y de los objetivos propuestos, así mismo declararon las necesidades y las barreras que quedaron por resolver, todo lo cual fue sustentado mediante diferentes evidencias, dentro de las que se destacaron los videos dando el testimonio de los padres en los que reconocían el trabajo práctico desarrollado por los estudiantes con sus hijos y los aprendizajes alcanzados por ellos en este proceso, a partir de las orientaciones recibidas de los estudiantes.

A partir de este informe se diseñaron las propuestas de mejora, los informes con las estrategias actualizadas a partir de los logros y las debilidades a resolver, fueron entregadas a los centros de prácticas para que sean aplicadas dando continuidad a los tratamientos. Durante esta etapa finalmente los estudiantes presentaron y sustentaron sus Proyectos integradores de saberes en los que expusieron mediante un informe todo el proceso de investigación acción desarrollado y los resultados alcanzados.

\section{Principales resultados}

Todos los estudiantes presentaron los PIENSA y el protocolo del trabajo de titulación, en los que se evidenció el desarrollo de un pensamiento científico, creativo e innovador en la construcción de su propio aprendizaje.

De los alumnos con trastornos en el lenguaje y habla atendidos por los estudiantes, fueron superados 8 que presentaban trastornos fonético-fonológicos.

Todos los alumnos atendidos avanzaron en el desarrollo de las diferentes áreas afectadas. 


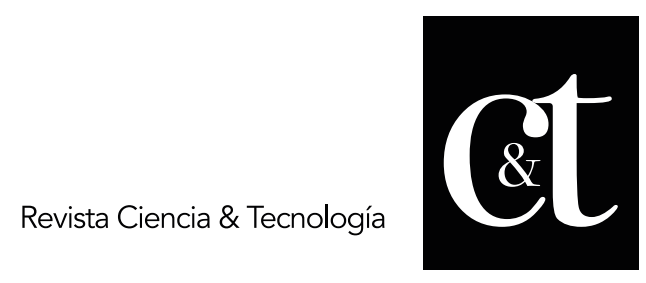

No. 32, 31 de octubre de 2021

ISSN impreso: 1390 - 6321

ISSN online: 2661 - 6734

Los estudiantes donaron material didáctico elaborados durante los tratamientos al Centro de Prácticas Inclusivas de la carrera.

Los estudiantes lograron desarrollar capacidades para realizar procesos de prevención, de evaluación-diagnóstico, y la intervención logopédica de los trastornos del habla y del lenguaje estudiados en estos ciclos, a partir de la práctica pre profesional desarrollada.

Desarrollaron capacidades para el diagnóstico y la intervención psicopedagógica integral desde un enfoque inclusivo que implicó la superación de las barreras en los contextos de aprendizaje social, familiar y escolar de los alumnos con trastornos logopédicos atendidos.

Se lograron los resultados de aprendizaje esperados en ambos ciclos, desarrollando las competencias profesionales y humanas expresadas en los conocimientos, las habilidades, las actitudes y valores de los estudiantes para comprender y contribuir a transformar las instituciones educativas hacia verdaderos procesos socioeducativos inclusivos.

\section{Conclusiones}

El estudio realizado evidenció el rol que desempeña hoy en el contexto ecuatoriano la UNAE en la formación de profesionales para atender los trastornos del lenguaje, habla, voz y comunicación en general, en las instituciones educativas regulares y especializadas de Ecuador, apoyando los procesos de aprendizaje y la inclusión socioeducativa, superando el enfoque clínico y la dispersión de la formación que aún prevalece en la especialidad; contribuyendo a la formación integral de la diversidad infantojuvenil de educandos en igual de oportunidades comprometidos con la transformación de la educación ecuatoriana.

Éste trabajo evidencia las experiencias vividas en la formación de los estudiantes del presente $7 \mathrm{mo}$ y $8 \mathrm{vo}$ ciclo en el itinerario de Logopedia de la carrera de Educación Especial de la UNAE, en un proceso académico que articula teoríapráctica mediante las prácticas pre profesionales y de servicios comunitario desarrolladas en el centro educativo especializado UDIPSAI, siguiendo la metodología investigación acción, logrando el desarrollo de capacidades para comprender, diagnosticar, diseñar y evaluar los trastornos del habla y del lenguaje estudiados y contribuir a la corrección de los trastornos fonético-fonológicos de los niños atendidos.

\section{Referencias bibliográficas}

González, H. Quinn, Á. Pérez Gómez, J. Prats, A. Didirksson, F. Peñafiel, \& S. Escribano, A. (2013) Inclusión educativa y profesorado inclusivo: aprender juntos para aprender a vivir juntos. Editorial Narcea

Bert, J; Naranjo, I; Rodríguez, A (2020) Las redes de apoyo en el contexto educativo escolar, familiar y comunitario: debate imprescindible para las prácticas educativas inclusivas. Revista Científica Ciencia Tecnología, Vol. 27. UTEG. 


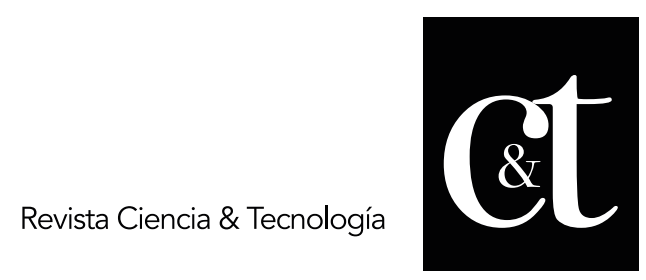

No. 32, 31 de octubre de 2021

ISSN impreso: 1390 - 6321

ISSN online: 2661 - 6734

Bert, J; Herrera, JI; Guevara G (2020) La formación de docentes para la inclusión socioeducativa. nuevos enfoques y perspectivas desde la universidad nacional de educación de ecuador (UNAE). Revista Científica Ciencia Tecnología. UTEG.

Herrera, J., Guevara, G., \& Bert, J. (2019). La formación de los docentes para la inclusión educativa en el marco del aula diversificada. Convergence Tech, 1(1), 105-121.

Herrera, JI; Parrilla, A; Blanco, A; Y Guevara Geysel (2018) Revista Latinoamericana de Educación Inclusiva. 12(1), 21-38

Pérez Gómez, A (2010) Aprender a educar: Nuevos desafíos para la profesión de docentes. Revista Interuniversitaria para la Formación del Profesorado

Actas del XXV Congreso de Logopedia, Foniatría y Audiología (2006) La logopedia en Iberoamérica. Granada, España. 